\section{Mortes evitáveis em menores de um ano, Brasil, 1997 a 2006: contribuições para a avaliação de desempenho do Sistema Único de Saúde}

\author{
Avoidable causes of infant mortality in Brazil, $1997-$ \\ 2006: contributions to performance evaluation of \\ the Unified National Health System
}

\begin{abstract}
Infant deaths were classified as avoidable, nonavoidable, and resulting from ill-defined conditions, from 1997 to 2006, using the Brazilian List of Avoidable Causes of Mortality. Non-linear regression was used to calculate trends in causespecific infant mortality rates. There was a significant decline in both avoidable deaths and deaths from ill-defined causes $(p<0.001)$. Avoidable deaths decreased by $37 \%$ overall. Mortality avoidable through adequate intrapartum care and adequate neonatal care decreased by $27.7 \%$ and $42.5 \%$, respectively, while mortality avoidable through adequate prenatal care increased by 28.3\%. In conclusion, health services contributed to the reduction in infant mortality. The decrease in ill-defined causes of death indicates expanded access to health services. The increase in access to intrapartum and neonatal care contributed to the reduction in infant deaths. The increase in mortality avoidable through adequate prenatal care indicates the need for improvement in prenatal care.
\end{abstract}

Infant Mortality; Cause of Death; Health Services Evaluation; Single Health System
Deborah Carvalho Malta 1,2 Elisabeth Carmen Duarte 3 Juan José Cortez Escalante 2 Márcia Furquim de Almeida 4 Luciana M. Vasconcelos Sardinha 2 Eduardo Marques Macário 2 Rosane Aparecida Monteiro 5 Otaliba Libânio de Morais Neto 2

\section{Introdução}

O conceito de morte evitável foi proposto inicialmente por Rutstein el al. ${ }^{1}$ como aquelas mortes que poderiam ter sido evitadas (em sua totalidade ou em parte) pela presença de serviços de saúde efetivos propondo uma lista com cerca de 90 causas. Inerente a essa e outras definições que se seguiram, está o fato de que determinados óbitos não devam ocorrer, por ser possível a prevenção e/ou o tratamento do evento ou da condição que o determina 2 .

Charlton \& Velez 3 foram os primeiros a aplicar o conceito de mortes evitáveis em estudos populacionais na Inglaterra e País de Gales. Posteriormente, estudos que aplicaram esse conceito se focaram especialmente na intervenção médica e noutras listas de causas de mortes evitáveis em conformidade com novos referenciais, avanços na intervenção médica e realidades locais 4 . Destacam-se os estudos de Holland 4, que estimularam pesquisas em diversos países e continentes (Europa, Ásia e Oceania).

De forma mais geral, este indicador é sensível à qualidade e diversidade da atenção à saúde prestada pelo sistema de saúde, sendo medida de resultado ou impacto dos serviços de saúde 4,5,6.

O conceito de morte evitável parece apropriado para o monitoramento e avaliação dos serviços de saúde, uma vez que a mensuração de indicadores de causas de morte evitáveis beneficia-se da objetividade, da oportunidade, da faci- 
lidade e da disponibilidade continuada de dados, permitindo, por exemplo, análises de tendências temporais e comparações entre regiões e municípios 6 . Além disso, segundo o referencial proposto por Habicht et al. 7, as análises de tendências de indicadores sensíveis à atuação do SUS podem fornecer, segundo o pressuposto da inferência de adequação, indícios sobre o desempenho desse sistema de saúde a fim de prevenir mortes desnecessárias e precoces da população.

Atualmente, existe uma extensa e crescente produção científica sobre evitabilidade de causas de morte no mundo e no Brasil, incluindo artigos de revisão 6,8 e avaliações empíricas 9. Além disso, existe a Lista Brasileira de Causas de Morte Evitáveis, segundo grupos etários de menores de cinco anos e de 5 a 74 anos, sob a perspectiva do SUS 10. A lista brasileira foi construída sob a coordenação da Secretaria de Vigilância em Saúde do Ministério da Saúde, envolvendo especialistas de áreas relevantes e intenso debate sobre o tema 10. O referencial teórico adotado na construção desta lista brasileira baseou-se em listas disponíveis no Brasil e no mundo e, em particular, nas listas de Ortiz ${ }^{11}$ e Tobias \& Jackson ${ }^{12}$. Essa análise considerou ainda os avanços atuais no campo da assistência à saúde e saúde pública no Brasil e o potencial de intervenção do SUS, conforme seus princípios e incorporação tecnológica atual 10.

Desde a publicação da Lista Brasileira de Causas de Morte Evitáveis, tem sido estimulada a sua utilização e validação, para o seu aprimoramento. Este artigo analisa a tendência de causas de mortes evitáveis em menores de um ano, com o objetivo de identificar mudanças que indiquem a adequação de uma hipótese de impacto do SUS 7. Além de discutir a utilidade da lista de mortes evitáveis por intervenções do SUS para análises dessa natureza.

\section{Metodologia}

Trata-se de estudo ecológico utilizando série temporal dos óbitos de menores de um ano de idade, ocorridos no Brasil e regiões, no período de 1997 a 200613.

O registro da causa de morte baseia-se na Classificação Internacional de Doenças, 10a Revisão (CID-10). A análise de tendência dos óbitos evitáveis neste período foi realizada com o emprego da Lista Brasileira de Mortes Evitáveis 10. Com base nesse referencial, as mortes foram classificadas em:

- Causas evitáveis: (a) reduzíveis pelas ações de imunização; (b) reduzível pela adequada atenção à mulher na gestação e parto e ao recém-nascido; (c) reduzíveis por ações adequadas de diagnós- tico e tratamento; e (d) reduzíveis por ações de promoção da saúde vinculadas a ações de atenção em saúde;

- Causas de mortes mal definidas;

- Causas de mortes não evitáveis (demais causas).

Foram analisados os seguintes indicadores: razão de mortalidade proporcional para menores de um ano e coeficiente de mortalidade infantil por: (i) causas evitáveis segundo grupos; (ii) mal definidas; e (iii) demais causas não evitáveis. Esses indicadores foram analisados para Brasil e regiões, no período de 1997 a 2006. Usualmente, não se calcula o coeficiente de mortalidade por causas mal definidas, porém este foi calculado para permitir melhor compreensão dos resultados obtidos.

Os coeficientes de mortalidade infantil foram calculados utilizando a metodologia proposta pela Rede Interagencial de Informações para a Saúde (RIPSA; http://tabnet.datasus.gov. br/cgi/tabcgi.exe?idb2007/c05.def, acessado em 10/Nov/2009), que combina dados diretos nas unidades federadas com adequada cobertura e regularidade nos Sistemas de Informação de Mortalidade (SIM) e Nascidos Vivos (SINASC) e dados indiretos nas demais Unidades da Federação. O presente estudo analisou a magnitude e tendências do coeficiente de mortalidade infantil mediante dados notificados no SIM e no SINASC. Sempre que possível foram utilizados os Coeficientes de Mortalidade Infantil (CMI) preconizados pelo Ministério da Saúde e RIPSA, combinando dados diretos (São Paulo, Rio de Janeiro, Espírito Santo, Mato Grosso do Sul, Distrito Federal, Paraná, Santa Catarina e Rio Grande do Sul) e dados indiretos (demais estados) com base em estimativas indiretas do Instituto Brasileiro de Geografia e Estatística (IBGE) e empregadas pela RIPSA. Como a RIPSA dispõe apenas de dados indiretos da mortalidade infantil, sem discriminação segundo causas, procedeu-se à distribuição do total de óbitos infantis estimados, obedecendo à mesma composição das causas observada nos dados diretos. Essa metodologia tem como pressuposto, que os óbitos não notificados apresentam a mesma distribuição proporcional, segundo causas dos óbitos notificados no SIM.

Foram estimadas as tendências temporais de 1997 a 2006 dos CMI segundo grupos de causas evitáveis pela análise de regressão não linear (modelo exponencial). O modelo não linear foi convertido à linear aplicando transformação logarítmica neperiana na variável dependente para normalizar a distribuição dos resíduos. Foram estimados os coeficientes da regressão brutos e ajustados pelo coeficiente de mortalidade por 
causas mal definidas. O programa Stata versão 9 (Stata Corp., College Station, Estados Unidos) foi utilizado nessa etapa da análise.

\section{Resultados}

No ano de 2006 foram notificados 47.629 óbitos de crianças menores de um ano de idade no Brasil, indicando uma redução de 33,3\% em relação ao ano de 1997. O número de óbitos por causas evitáveis também caiu no mesmo período, passando de 50.952 em 1997, para 33.064 em 2006, expressando uma redução de 17.888 óbitos (Tabela 1).

A Tabela 1 mostra que nesse período observou-se uma redução de $35,2 \%$ da CMI no país. O coeficiente de mortalidade por causas de morte evitáveis teve uma redução de $37 \%$ no período estudado, o coeficiente de mortalidade por causas mal definidas apresentou um declínio de $75,7 \%$ e as demais causas de morte, um declínio de $2,2 \%$. A redução das causas mal definidas também se expressa no declínio da proporção de óbitos passando de 11,2\% em 1997 para 4,2\% em 2006, no Brasil.

A Tabela 1 mostra os coeficientes de mortalidade para os grupos de causas incluindo as principais causas de morte específicas para cada grupo de causas. No grupo das causas reduzíveis por ações de imunização, foram observados 189 óbitos em 1997 e 46 óbitos em 2006, sendo nesse último ano: 20 óbitos por coqueluche, 9 por tuberculose, 6 por tétano neonatal, 10 por hepatite e 1 por meningite por haemophilus. A redução do coeficiente de mortalidade deste grupo foi de $75 \%$ no período estudado.

O CMI por ações de promoção à saúde vinculada a ações de atenção em saúde reduziu em $56,5 \%$ entre 1997 e 2006 . As causas que mais contribuem com esse grupo são as doenças infecciosas intestinais com redução de $66,7 \%$ do coeficiente de mortalidade. As deficiências nutricionais também apresentaram declínio expressivo do coeficiente de mortalidade $57,4 \%$ (Tabela 1). Além disso, merecem destaque também as causas externas, em especial outros riscos acidentais à respiração (750 óbitos em 1997 e 504 óbitos em 2006), eventos cuja intenção é indeterminada (127 óbitos em 1997 e 125 óbitos em 2006) e acidentes de transporte (168 óbitos em 1997 e 102 óbitos em 2006) (dados não apresentados).

Houve uma redução de $47,7 \%$ do coeficiente de mortalidade das causas reduzíveis por ações adequadas de diagnóstico e tratamento. As causas que mais contribuem com esse grupo são as pneumonias com redução de $52,7 \%$ do seu coeficiente específico de mortalidade (Tabela 1).
Cumpre destacar também a meningite - doença do sistema nervoso central, exceto G000 - (704 óbitos em 1997 e 267 óbitos em 2006) e outras infecções agudas das vias aéreas inferiores (309 óbitos em 1997 e 208 óbitos em 2006), dados não apresentados.

O CMI específico das causas reduzíveis por adequada atenção prestada ao recém-nascido apresentou um declínio de 42,5\%. Observou-se uma redução de $40,6 \%$ do coeficiente de mortalidade por desconforto respiratório do recémnascido, os transtornos respiratórios e cardiovasculares do recém-nascido declinaram em $75,6 \%$, já as infecções específicas do período perinatal apresentaram redução de apenas $2 \%$ (Tabela 1).

As causas reduzíveis por adequada atenção à mulher no parto apresentaram uma redução de $27,7 \%$. As causas que mais contribuíram com este grupo de causa de morte foram: hipoxia intra-uterina e asfixia ao nascer, que apresentaram uma redução de $28,7 \%$, e aspiração neonatal com declínio de 39,5\% (Tabela 1).

Por outro, lado chama a atenção o aumento de $28,3 \%$ das causas de morte relacionadas à adequada atenção à mulher na gestação. Houve um crescimento de $28,2 \%$ do coeficiente de mortalidade dos transtornos relacionados à gestação de curta duração e baixo peso ao nascer. Verificou-se um aumento de $100 \%$ do coeficiente de mortalidade das afecções maternas que afetam o feto ou o recém-nascido e de $27 \%$ das complicações maternas da gravidez que afetam o feto ou o recém-nascido (Tabela 1).

No mesmo período de análise no Brasil, as demais causas de morte infantil que foram consideradas como não evitáveis mostraram certa estabilidade, partindo de 12.438 óbitos (5,6 óbitos por mil nascidos vivos) em 1997, para 12.550 (5,4 óbitos por mil nascidos vivos) em 2006, ou redução de 2,2\% no CMI no período.

Os resultados da análise de tendência temporal no período de 1997 a 2006 dos CMI estimados pelos modelos de regressão concordam com os resultados descritos anteriormente na Tabela 1. Tal análise, no entanto, permite ainda o ajuste das tendências dos CMI (por causas evitáveis ou não) pela redução importante observada no CMI por causas mal definidas (Tabela 2). A redução média (bruta) do CMI por causas mal definidas foi de $14,6 \%$ por ano [efeito $=(0,854-1)$, ou efeito de -0,158 no log (CMI por causas mal definidas)] entre 1997 e 2006. A redução média (bruta) do CMI das causas evitáveis foi de 5\% por ano [efeito $=(0,950-1)$, ou efeito de $-0,051$ no $\log (\mathrm{CMIev})]$ e de $6,9 \%$ por ano, quando ajustada pela tendência de redução do CMI por causas mal definidas, considerando o mesmo período de análise (Tabela 2). 
Tabela 1

Número de óbitos e coeficiente de mortalidade infantil (CMI), por mil nascidos vivos, segundo principais causas reduzíveis e demais causas de morte. Brasil, $1997,2000,2003$ e 2006.

\begin{tabular}{|c|c|c|c|c|c|c|c|c|c|}
\hline \multirow[t]{2}{*}{ Causas de morte } & \multicolumn{2}{|c|}{1997} & \multicolumn{2}{|c|}{2000} & \multicolumn{2}{|c|}{2003} & \multicolumn{2}{|c|}{2006} & \multirow{2}{*}{$\begin{array}{c}\text { Variação } \\
\text { percentual } \\
(2006 / 1997)\end{array}$} \\
\hline & $\mathbf{n}$ & CMI & $\mathrm{n}$ & CMI & $\mathrm{n}$ & CMI & $\mathbf{n}$ & CMI & \\
\hline Causas reduzíveis/evitáveis & 50.952 & 22,76 & 46.509 & 18,66 & 39.661 & 16,46 & 33.064 & 14,34 & $-37,0$ \\
\hline Ações de imunização & 189 & 0,08 & 109 & 0,04 & 68 & 0,03 & 46 & 0,02 & $-75,0$ \\
\hline Coqueluche & 16 & 0,01 & 27 & 0,01 & 19 & 0,01 & 20 & 0,01 & 0,0 \\
\hline Tuberculose & 45 & 0,02 & 28 & 0,01 & 22 & 0,01 & 9 & 0,00 & $-100,0$ \\
\hline Demais causas do grupo & 128 & 0,06 & 54 & 0,02 & 27 & 0,01 & 17 & 0,01 & $-83,3$ \\
\hline $\begin{array}{l}\text { Adequada atenção à mulher na } \\
\text { gestação }\end{array}$ & 4.738 & 2,12 & 5.195 & 2,08 & 5.351 & 2,22 & 6.282 & 2,72 & 28,3 \\
\hline $\begin{array}{l}\text { Transtornos relacionados à } \\
\text { gestação de curta duração e baixo } \\
\text { peso ao nascer }\end{array}$ & 2.774 & 1,24 & 2.794 & 1,12 & 3.147 & 1,31 & 3.675 & 1,59 & 28,2 \\
\hline $\begin{array}{l}\text { Afecções maternas que afetam o } \\
\text { feto ou o recém-nascido }\end{array}$ & 555 & 0,25 & 758 & 0,30 & 770 & 0,32 & 1.150 & 0,50 & 100,0 \\
\hline $\begin{array}{l}\text { Complicações maternas da } \\
\text { gravidez que afetam o feto ou o } \\
\text { recém-nascido }\end{array}$ & 821 & 0,37 & 1.067 & 0,43 & 962 & 0,40 & 1.081 & 0,47 & 27,0 \\
\hline Demais causas do grupo & 588 & 0,26 & 576 & 0,23 & 472 & 0,20 & 376 & 0,16 & $-38,5$ \\
\hline Adequada atenção à mulher no parto & 5.918 & 2,64 & 5.560 & 2,23 & 4.736 & 1,97 & 4.406 & 1,91 & $-27,7$ \\
\hline $\begin{array}{l}\text { Hipoxia intra-uterina e asfixia ao } \\
\text { nascer }\end{array}$ & 3.834 & 1,71 & 3.597 & 1,44 & 3.119 & 1,29 & 2.807 & 1,22 & $-28,7$ \\
\hline Aspiração neonatal & 1.806 & 0,81 & 1.545 & 0,62 & 1.239 & 0,51 & 1.120 & 0,49 & $-39,5$ \\
\hline Demais causas do grupo & 278 & 0,12 & 418 & 0,17 & 378 & 0,16 & 479 & 0,21 & 75,0 \\
\hline Adequada atenção ao recém-nascido & 25.330 & 11,32 & 23.579 & 9,46 & 19.490 & 8,09 & 15.004 & 6,51 & $-42,5$ \\
\hline $\begin{array}{l}\text { Transtornos respiratórios e } \\
\text { cardiovasculares específicos do } \\
\text { período neonatal }\end{array}$ & 9.082 & 4,06 & 7.756 & 3,11 & 4.953 & 2,06 & 2.289 & 0,99 & $-75,6$ \\
\hline $\begin{array}{l}\text { Infecções específicas do período } \\
\text { perinatal }\end{array}$ & 5.690 & 2,54 & 5.651 & 2,27 & 5.505 & 2,28 & 5.734 & 2,49 & $-2,0$ \\
\hline $\begin{array}{l}\text { Desconforto respiratório do } \\
\text { recém-nascido }\end{array}$ & 7.124 & 3,18 & 6.584 & 2,64 & 5.846 & 2,43 & 4.355 & 1,89 & $-40,6$ \\
\hline Demais causas do grupo & 3.434 & 1,53 & 3.588 & 1,44 & 3.186 & 1,32 & 2.626 & 1,14 & $-25,5$ \\
\hline $\begin{array}{l}\text { Ações de diagnóstico e tratamento } \\
\text { adequados }\end{array}$ & 7.836 & 3,50 & 6.426 & 2,58 & 5.248 & 2,18 & 4.214 & 1,83 & $-47,7$ \\
\hline Pneumonia & 3.779 & 1,69 & 3.008 & 1,21 & 2.438 & 1,01 & 1.837 & 0,80 & $-52,7$ \\
\hline Outras doenças bacterianas & 2.197 & 0,98 & 1.899 & 0,76 & 1.572 & 0,65 & 1.217 & 0,53 & $-45,9$ \\
\hline Demais causas do grupo & 1.860 & 0,83 & 1.519 & 0,61 & 1.238 & 0,51 & 1.160 & 0,50 & $-39,8$ \\
\hline $\begin{array}{l}\text { Ações de promoção à saúde } \\
\text { vinculadas a ações de atenção }\end{array}$ & 6.941 & 3,10 & 5.641 & 2,26 & 4.768 & 1,98 & 3.112 & 1,35 & $-56,5$ \\
\hline Doenças infecciosas intestinais & 4.035 & 1,80 & 3.004 & 1,21 & 2.387 & 0,99 & 1.384 & 0,60 & $-66,7$ \\
\hline Deficiências nutricionais & 1.515 & 0,68 & 1.385 & 0,56 & 1.259 & 0,52 & 667 & 0,29 & $-57,4$ \\
\hline Demais causas do grupo & 1.391 & 0,62 & 1.252 & 0,50 & 1.122 & 0,47 & 1.061 & 0,46 & $-25,8$ \\
\hline Causas mal definidas & 8.010 & 3,58 & 8.388 & 3,37 & 4.804 & 1,99 & 2.015 & 0,87 & $-75,7$ \\
\hline Todas as demais causas de morte & 12.438 & 5,56 & 13.304 & 5,34 & 13.077 & 5,43 & 12.550 & 5,44 & $-2,2$ \\
\hline Total & 71.400 & 31,90 & 68.199 & 27,36 & 57.540 & 23,88 & 47.629 & 20,66 & $-35,2$ \\
\hline
\end{tabular}

Fonte: Sistema de Informações sobre Mortalidade (SIM), Sistema de Informações de Nascidos Vivos (SINASC) e Rede Interagencial de Informações para a Saúde (RIPSA). 
Regressão exponencial do coeficiente de mortalidade infantil (CMI), por mil nascidos vivos, segundo grupos de causas evitáveis, mal definidas e demais causas. Brasil, 1997 a 2006.

\begin{tabular}{|c|c|c|c|c|}
\hline Variável dependente & Coeficiente (IC95\%) * & $\begin{array}{c}\text { Exponencial } \\
\text { [Coeficiente (IC95\%)] }\end{array}$ & Valor de $p$ & $\mathbf{R}^{2}$ \\
\hline \multicolumn{5}{|l|}{ CMI por causas evitáveis } \\
\hline Bruta & $-0,051(-0,046 ;-0,056)$ & $0,950(0,955 ; 0,946)$ & $<0,001$ & 0,9849 \\
\hline Ajustado por causas mal definidas & $-0,072(-0,047 ;-0,097)$ & $0,931(0,954 ; 0,908)$ & $<0,001$ & 0,9904 \\
\hline \multicolumn{5}{|l|}{$\mathrm{CMI}$ por demais causas } \\
\hline Bruta & $-0,003(-0,007 ; 0,001)$ & $0,997(0,993 ; 1,001)$ & 0,135 & 0,2564 \\
\hline Ajustado por causas mal definidas & $-0,023(-0,005 ;-0,042)$ & $0,977(0,995 ; 0,959)$ & $<0,020$ & 0,6304 \\
\hline CMI por causas mal definidas & $-0,158(-0,125 ;-0,190)$ & $0,854(0,882 ; 0,827)$ & $<0,001$ & 0,9392 \\
\hline
\end{tabular}

* Log: Logaritmo neperiano.

Fonte: Sistema de Informações sobre Mortalidade (SIM), Sistema de Informações de Nascidos Vivos (SINASC) e Rede Interagencial de Informações para a Saúde (RIPSA).

Essa redução foi marcadamente superior à redução do coeficiente de mortalidade das demais causas morte (não classificadas como evitáveis) no mesmo período. A redução média (bruta) do CMI por essas demais causas foi de $0,3 \%$ por ano [efeito $=(0,997-1)$, ou efeito bruto de -0,003 no log (CMI pelas demais causas)], resultado não significante estatisticamente. Essa tendência eleva-se em $0,3 \%$ por ano, quando ajustada pela tendência de redução do CMI por causas mal definidas (Tabela 2). Todas as tendências ajustadas pelo CMI das causas mal definidas foram estatisticamente significantes $(\mathrm{p}<0,05)$

Os resultados do coeficiente de mortalidade por causas reduzíveis segundo regiões do Brasil mostram que a redução mais acentuada se deu na Região Sudeste $(42 \%)$, seguida da Região Centro-Oeste $(36,5 \%)$, Nordeste $(32,4 \%)$ e Sul (32,4\%), período de 1997 a 2007. Na Região Norte a redução foi mais discreta para o período de 1997 e 2007 (28,6\%) (Tabela 3).

\section{Discussão}

O declínio do coeficiente de mortalidade infantil no período estudado foi de $35,2 \%$, semelhante ao encontrado em outros estudos 14,15. O resultado é consistente com outros estudos que mostram a tendência de declínio da mortalidade infantil, ao longo do tempo 16,17 . Simões 16 indica que a atuação dos serviços de saúde contribuiu de forma importante para a redução da mortalidade infantil no país e sugere que frente ao desempenho negativo de vários indicadores sociais na década 1990 a atuação dos serviços de saúde permitiu que este indicador mantivesse a tendência de declínio. Outro estudo realizado entre 1980 e 2000 identificou que a queda da taxa de fecundidade foi um fator determinante para o declínio da mortalidade infantil no país, contudo indica também que as ações básicas de saúde e sua interiorização contribuíram para o declínio do CMI 17.

Este é um estudo descritivo que pretende analisar o comportamento das causas de morte passíveis de redução pela ação dos serviços de saúde, não se pretende identificar qual foi o papel dos serviços de saúde na cadeia causal da mortalidade infantil. Entretanto, os resultados permitiram observar que houve uma atuação positiva dos serviços de saúde no perfil de mortalidade infantil do país. As listas de causas de morte consideradas como potencialmente evitáveis ou reduzíveis por conta da ação dos serviços de saúde permitem identificar em quais causas de morte foi possível obter ganhos ao longo do tempo e em quais situações há necessidade de investir em uma atuação mais adequada.

Segundo Malta \& Duarte 6, o emprego de listas de causas de morte constitui-se em importante instrumento de avaliação de desempenho dos serviços de saúde. Isso porque é esperado que a morte evitável seja sensível, em algum grau, à qualidade da atenção à saúde prestada pelo sistema de saúde. São freqüentes os aprimoramentos nas listas de mortes evitáveis usadas pelos diferentes autores, refletindo especificidades locais, avanços na atenção à saúde, bem como novos conceitos incorporados mediante processos de debate e reflexão. Por exemplo, internacionalmente as tabelas de evitabilidade, sobretudo as baseadas na proposta de Rutstein 
Tabela 3

Coeficiente de mortalidade infantil (CMI), por mil nascidos vivos, por causas evitáveis. Brasil e regiões, 1997-2006.

\begin{tabular}{|c|c|c|c|c|c|c|c|c|c|c|c|}
\hline Região & 1997 & 1998 & 1999 & 2000 & 2001 & 2002 & 2003 & 2004 & 2005 & 2006 & $\begin{array}{c}\text { Variação } \\
\text { percentual } \\
(2006 / 1997)\end{array}$ \\
\hline Norte & 22,3 & 21,6 & 21,1 & 20,0 & 19,4 & 18,1 & 17,7 & 16,6 & 16,2 & 15,9 & $-28,6$ \\
\hline Nordeste & 32,4 & 30,4 & 28,2 & 26,5 & 26,5 & 25,4 & 24,1 & 22,9 & 22,7 & 21,4 & $-34,0$ \\
\hline Sudeste & 17,5 & 16,3 & 14,4 & 13,6 & 12,8 & 12,1 & 11,7 & 11,1 & 10,5 & 10,2 & $-42,0$ \\
\hline Sul & 12,8 & 13,1 & 12,2 & 12,0 & 11,3 & 11,0 & 10,7 & 10,1 & 9,15 & 8,64 & $-32,4$ \\
\hline Centro-Oeste & 18,0 & 17,0 & 15,6 & 14,8 & 14,7 & 13,5 & 13,2 & 12,7 & 12,1 & 11,4 & $-36,5$ \\
\hline Brasil & 22,8 & 21,4 & 19,5 & 18,7 & 18,2 & 17,2 & 16,5 & 15,5 & 15,0 & 14,3 & $-37,3$ \\
\hline
\end{tabular}

Fonte: Sistema de Informações sobre Mortalidade (SIM), Sistema de Informações de Nascidos Vivos (SINASC) e Rede Interagencial de Informações para a Saúde (RIPSA).

et al. 1, incluíam todos os subcomponentes da mortalidade infantil. Em função da redução importante da mortalidade infantil nos países do primeiro mundo, pesquisadores desses países passaram a adotar a mortalidade perinatal como foco de monitoramento e redefiniram as listas de acordo com essa proposta 4,18.

O componente neonatal é o predominante na mortalidade infantil do país ${ }^{14}$, contudo as desigualdades de perfil epidemiológico justificam a inclusão de causas de morte mais freqüentemente encontradas no período pós-neonatal como, por exemplo, as doenças infecciosas intestinais e pneumonias nas listas brasileiras de causas de morte evitáveis. Assim, a lista adotada no presente estudo e preconizada pelo Ministério da Saúde 10 inclui estas causas de morte.

Houve uma substancial redução $(75,7 \%)$ da taxa de mortalidade por causas mal definidas no período de 1997 a 2006. Usualmente não se calcula o coeficiente de mortalidade por causas mal definidas, mas neste caso foi calculado com o objetivo de melhor identificar seu comportamento ao longo do tempo. O resultado indica, de um lado, que houve maior acesso aos serviços de saúde possibilitando a identificação da causa de morte e, de outro, a melhoria da gestão do SIM, buscando qualificar melhor as informações disponíveis.

Em locais em que as causas mal definidas apresentam elevada participação relativa na mortalidade, o emprego de listas de causas de morte evitáveis pode ficar comprometido. Usualmente, considera-se uma informação de boa qualidade quando os ignorados ou não informados situamse abaixo de $10 \%$, de modo a não comprometer a distribuição de valores ou categorias conhecidas 19. Os resultados obtidos mostram que as causas mal definidas representavam $10,8 \%$ do total de óbitos infantis em 1997, situando-se assim 0,8\% acima do que se considera uma informação de boa qualidade. Em 2006 os óbitos por causas mal definidas representavam $4,2 \%$ do total de óbitos infantis. Algumas distorções podem estar presentes na análise da tendência de mortalidade por causas evitáveis, pois se assumiu que as causas de morte mal definidas teriam distribuição semelhante às demais conhecidas ao longo do tempo. Entretanto, o declínio das causas mal definidas indica, juntamente com restante dos resultados, que ocorreu uma ação positiva dos serviços de saúde em relação aos óbitos infantis.

As causas de morte consideradas como reduzíveis pela ação de serviços de saúde mostraram um declínio de $37 \%$, enquanto as causas não incluídas na lista de evitabilidade tiveram no período em estudo um discreto decréscimo de $2,2 \%$, que não foi estatisticamente significante. Como decorrência, constatou-se que aconteceu um crescimento da participação relativa dos óbitos por causas não evitáveis no conjunto de óbitos de menores de um ano.

Esses resultados podem gerar várias hipóteses explicativas. Inicialmente, por se tratar de causas que teoricamente são sensíveis à atenção à saúde oportuna e de boa qualidade, pode ser levantada a hipótese de que o Brasil vem experimentando melhoria no desempenho do SUS. Tal hipótese se sustenta no notório aprimoramento de oferta, cobertura e utilização das ações e serviços vinculados ao SUS 14,15,20. Estudos internacionais mostram benefícios dos fatores assistenciais na redução dos óbitos evitáveis, apontando diferentes aspectos dos sistemas de saúde que interferem na redução da mortalidade 3,18,21. Mesmo com diferenças metodológicas, esses estudos apontam que as causas não evitáveis declinam mais lentamente que as evitáveis. 
Os resultados obtidos apresentaram comportamento semelhante.

Dentre os grupos de causas evitáveis o grupo de causas reduzíveis por imunização apresentou uma redução de $75 \%$ no período de estudo. O grupo de causas reduzíveis por ações de promoção à saúde vinculadas a ações de atenção apresentaram o segundo maior declínio (56,5\%), seguido pelo grupo de causas reduzíveis por ações adequadas de diagnóstico e tratamento $(47,7 \%)$ e do grupo de causas associado à adequada atenção ao recém-nascido (42,5\%). Esses resultados mostram o impacto positivo dos serviços de saúde sobre os óbitos infantis.

Outra hipótese, não excludente da anterior, é a contribuição paralela da melhoria de condições de vida da população brasileira. As causas de morte reduzíveis são sensíveis à atenção à saúde, mas elas também respondem de maneira importante à melhoria das condições de vida, acesso a bens e serviços, escolaridade e renda, entre outros determinantes sociais do processo saúdedoença, como apontado por alguns autores 22,23. No Brasil, nos últimos anos, marcante desenvolvimento econômico e social tem sido observado, o que pode ser evidenciado, por exemplo, pelo incremento da escolaridade e redução das desigualdades sociais 20 (http://tabnet.datasus.gov. br/cgi/tabcgi.exe?idb2007/c05.def, acessado em 10/Nov/2009).

Com relação ao grupo de causas reduzíveis por ações de imunoprevenção, é necessário algum cuidado na interpretação do elevado declínio $(75 \%)$ apresentado. Este coeficiente de mortalidade é pequeno e qualquer oscilação de valor pode apresentar grande variação. No entanto, os baixos valores dos coeficientes de mortalidade por estas causas de morte também mostram o sucesso do PNI (Programa Nacional de Imunizações) no país. Outros estudos já mostravam a importância do PNI na redução da moralidade infantil 16,17. Contudo, verifica-se que ainda poderão ocorrer ganhos futuros, pois, ainda que em número reduzido, foram registrados óbitos infantis por coqueluche e tuberculose em 2006.

No grupo de causas reduzíveis por ações de promoção e adequada atenção à saúde, destacase o consistente declínio da taxa de mortalidade por doenças infecciosas intestinais $(66,7 \%)$. Outros estudos já haviam mostrado o efeito positivo da expansão de saneamento básico na mortalidade e incidência dessas doenças 14,22. Neste período ocorreu um aumento da proporção de domicílios ligados à rede de água encanada que era de 76,2\% em 1997 e 81,7\% em 2006 (http:// tabnet.datasus.gov.br/cgi/tabcgi.exe?idb2007/ c05.def, acessado em 10/Nov/2009). Porém, o crescimento seria insuficiente para explicar a redução do risco de morte por tais causas. Possivelmente este declínio também está associado à inclusão da TRO (Terapia de Reidratação Oral) entre as ações básicas de saúde desenvolvidas pelas Equipes de Saúde da Família na redução da letalidade destas doenças 24 .

Entre as causas reduzíveis por adequado diagnóstico e tratamento, destacam-se as pneumonias que apresentaram uma redução de 48,6\%. A redução possivelmente se deve ao maior acesso ao diagnóstico precoce de doenças respiratórias agudas e ao pronto tratamento dessas afecções, sugerindo que a expansão da rede básica de atenção à saúde e da estratégia do programa de saúde da família 15 podem ter contribuído para o resultado. Cabe lembrar também que a maior facilidade de acesso a internações pelo SUS pode ter resultado na redução da letalidade dessas afecções.

A redução das causas de morte acima mencionadas e da mortalidade por deficiências nutricionais $(57,4 \%)$ possa também estar relacionada à redução da incidência dessas doenças. Estas causas de morte estão associadas a precárias condições de vida. Verificou-se que vários indicadores sociais apresentaram um desempenho positivo no país no período, como por exemplo, houve redução da taxa de analfabetismo que passou de $14,7 \%$ em 1997 para 10,4\% em 2006, redução da proporção de pobres que era de $42,2 \%$ em 1997 e em 2006 34\% (http://tabnet.datasus. gov.br/cgi/tabcgi.exe?idb2007/c05.def, acessado em 10/Nov/2009).

Com relação ao conjunto de causas reduzíveis por adequada atenção à saúde, observa-se que há um comportamento diferenciado entre os subgrupos deste conjunto. Ocorreu expressiva redução de óbitos e da taxa de mortalidade por adequada atenção ao recém-nascido e do subgrupo de causas reduzíveis por adequada atenção ao parto no período em estudo, indicando que aconteceu uma melhora na atenção ao parto e ao recém nascido. No entanto, existiu aumento $(28,6 \%)$ das taxas de mortalidade das causas de morte reduzíveis por adequada atenção à gestação.

A redução da mortalidade por adequada atenção ao parto é consistente com a evolução de indicadores que mostram ter ocorrido crescente institucionalização da atenção ao parto em todo o país. Dados provenientes do SINASC mostram que a proporção de nascidos vivos em hospitais representava 97,2\% em 2006 no Brasil 14. É possível que os dados do SINASC superestimem os nascimentos hospitalares, pois há possibilidade de ocorrer menor notificação de nascimentos domiciliares ou é possível que nascimentos ocorridos no domicílio recebam pos- 
terior assistência hospitalar e sejam notificados como hospitalares 25. Contudo, esses resultados são consistentes com os dados da PNDS (Pesquisa Nacional de Demografia e Saúde) que mostram redução de nascimentos domiciliares 26 .

Encontrou-se também redução de óbitos por causas perinatais, como a aspiração neonatal $(39,5 \%)$ e hipoxia intra-uterina e asfixia ao nascer (28,6\%), que pode estar associada à ampliação e melhoria do acesso à atenção ao parto. Vale lembrar que a asfixia ao nascer e hipoxia intra-uterina são manifestações sindrômicas que podem ser decorrentes de outras afecções, e sua redução pode ser decorrente da melhor indicação de outras causas de morte.

O CMI por causas reduzíveis por adequada atenção ao recém-nascido apresentou uma redução de 42,5\%, representando uma redução de cerca de 10 mil óbitos no período de dez anos, corroborando para a indicação da ampliação do acesso à atenção ao parto e ao recém-nascido. Mas há um comportamento diferenciado dos subgrupos deste conjunto de óbitos. Encontrouse elevada redução do coeficiente de mortalidade por transtornos respiratórios e cardiovasculares e por desconforto respiratório do recém-nascido. Os dados indicam que as melhorias dos cuidados com os recém-nascidos logo após o parto possivelmente se devam ao aumento de acesso a cuidados intensivos neonatais, entre os quais a introdução do uso de surfactante no SUS. No entanto, os óbitos por infecções específicas no período perinatal mantiveram-se praticamente estáveis, demonstrando a necessidade de monitoramento da atenção prestada em berçários e unidades de terapia intensiva neonatal 27,28.

Na contramão da redução do coeficiente de mortalidade dos demais grupos de causas de morte reduzíveis devido à atuação dos serviços de saúde, ocorreu um aumento $(28,6 \%)$ dos coeficientes de mortalidade das causas de morte reduzíveis por adequada atenção à gestação. Essa tendência contradiz em parte os resultados do SINASC que mostram ter ocorrido uma ampliação do acesso à atenção pré-natal no país nos últimos anos 14 com redução da proporção de nascimentos que não haviam recebido assistência pré-natal. Em 1997, a proporção de nascimentos sem assistência pré-natal era de $6,7 \%$, caindo para $2,2 \%$ em 2006. A proporção de nascimentos para os as mães haviam realizado 7 ou mais consultas era de $41,6 \%$ em 1997 e de $54,5 \%$ em 2006 14. Esses dados mostram uma tendência de ampliação da assistência pré-natal no país, por mais que existam problemas relacionados à qualidade dessa oferta. É possível que a expansão da oferta da atenção pré-natal tenha resultado em diagnósticos mais efetivos da presença de do- enças e de complicações da/na gestação, resultando também em uma melhor identificação das causas de morte associadas à presença de afecções maternas. Contudo, estudos realizados em Pelotas, Campinas e São Paulo 27,28,29 mostraram que assistência pré-natal inadequada é um dos fatores de risco mais importantes na mortalidade neonatal. Esses resultados sugerem que não basta ampliar o acesso, mas é necessário investir na melhoria da qualidade da assistência pré-natal.

Fazem parte do conjunto de óbitos reduzíveis por adequada atenção à gestação os óbitos decorrentes de afecções maternas que afetam o recém-nascido. Esses óbitos praticamente dobraram no período em estudo. Neste grupo encontra-se a presença de diabetes, doenças renais e outras afecções maternas. É possível que a ampliação da assistência pré-natal tenha contribuído para o melhor diagnóstico dessas afecções na gestação como foi mencionado anteriormente. Entretanto, pode ter ocorrido também um aumento da prevalência dessas afecções. A primeira hipótese parece ser a mais provável, já que se verificou uma redução dos óbitos infantis por hipoxia e asfixia que podem ser decorrentes da presença dessas afecções.

Houve também um aumento do número de óbitos infantis por conta de complicações maternas da gestação; entre tais complicações, especial importância deve ser dada à hipertensão e à eclampsia, que em grande parte poderiam ter sido melhor manejadas no pré-natal. Estas afecções se constituem nos principais fatores de risco para a mortalidade neonatal precoce 29 . A ampliação do acesso à atenção pode ter resultado na melhor identificação desses óbitos, porém esta ampliação mostrou-se insuficiente para evitar a ocorrência de óbitos por essas causas.

Entre o grupo de causas reduzíveis por adequada atenção àgestação, encontram-se os óbitos por transtornos relacionados à gestação de curta duração e baixo peso ao nascer, que apresentaram um crescimento de $28,2 \%$ do coeficiente de mortalidade no período estudado. A etiologia desses nascimentos é complexa e envolve fatores sócio-econômicos, características maternas e reprodutivas, bem como a qualidade da atenção pré-natal 28,30. Alguns estudos mostram uma tendência de crescimento dos nascimentos de pré-termo em algumas cidades brasileiras, como Ribeirão Preto e Pelotas 31,32. O SINASC registrou aumento do registro de recém-nascidos de baixo peso de 7,7\% em 1997 para 8,2\% em 2006 e de nascimentos de pré-termo de 5,1\% em 1997 para $6,6 \%$ em 2006. O aumento pode ter sido ainda maior, pois é possível que ocorra o sub-registro de nascimentos de muito pré-termo no SINASC e o formato de preenchimento, por categorias de 
duração da gestação (32 a 36 semanas, 37 semanas e mais) 14,33 também pode contribuir para a subnotificação.

Os resultados mostram que houve um declínio das causas de morte potencialmente reduzíveis pela ação dos serviços de saúde em todas as regiões do país, entretanto este declínio foi desigual. Verificou-se que a redução mais acentuada ocorreu na Região Sudeste (45,5\%). Este resultado pode ser decorrente da concentração de serviços de saúde na Região Sudeste, mas também refletir desigualdades sociais regionais, que podem estar associadas à maior incidência de determinadas doenças, influindo indiretamente nos resultados. Alguns autores mostram que as variações geográficas das causas reduzíveis podem estar relacionadas não à melhoria dos serviços de saúde e sim à melhoria de outros fatores sócio-econômicos. Esses autores discutem o fato de que as evidências de associação geográficas de redução de mortalidade e serviços de saúde são fracas e inconsistentes 18 .

Os resultados obtidos mostraram importante redução dos óbitos por causas mal definidas no período de analise. Há, todavia, uma distribuição regional desigual desses óbitos, ocorrendo uma concentração na Região Norte e Nordeste. Também se observa um diferencial regional da cobertura ou captação de óbitos pelo SIM. Essas limitações devem ser consideradas em análises de causas de óbitos evitáveis comparando as diferentes regiões do Brasil 14.

Outros limites estão presentes no emprego das listas de causas de morte potencialmente evitáveis ou reduzíveis, e deve-se trabalhar inicialmente com a perspectiva de validação das listas, indagando em primeiro lugar se de fato as causas propostas podem ser impactadas pelas ações de atenção à saúde. Outro limite do estudo refere-se à análise dos determinantes externos à atenção à saúde que podem afetar a ocorrência dos eventos analisados. Por exemplo, a identificação de associações ecológicas das mortes evitáveis com a melhoria da qualidade e cobertura da atenção à saúde deve ser interpretada com cuidado, considerando os outros fatores que podem alterar a incidência e a letalidade dessas causas de morte independente do setor saúde, o que pode ocorrer em função da mudança dos fatores de risco, particularmente quando na análise de períodos prolongados 6,34.

Mesmo considerando as dificuldades metodológicas acima apontadas, os indicadores de evitabilidade podem ser importantes indicadores de efetividade dos serviços de saúde, e seu monitoramento é de grande valia para a análise de desempenho desses serviços em uma região ou país 6,7 .

Este trabalho se constitui em uma análise inicial do uso da lista de mortes de causas evitáveis no Brasil. Deverão ser realizados estudos futuros abordando diferenciais das taxas de mortalidade por causas evitáveis segundo grupos etários assim como segundo regiões e municípios do Brasil. Importante ainda explorar a melhor maneira de incorporação da variável "peso ao nascer" para a classificação da evitabilidade dos óbitos infantis. Esforços devem ainda ser focalizados na feitura de estudos de validação da lista de evitabilidade e na sua adaptação às diferentes realidades locais. Cumpre salientar ainda a necessidade de se conhecer e ampliar a validade da causa de morte infantil notificada nas declarações de óbito, o que pode interferir em análises dessa natureza, como as apresentadas no presente estudo. Finalmente, etapas seguintes desse processo envolvem ainda a atribuição de peso aos diferentes níveis de atenção (primária, secundária e terciária) envolvidos na evitabilidade dessas mortes em menores de um ano de idade, o que poderia auxiliar sobremaneira a definição de estratégias de intervenção oportunas e efetivas para a prevenção do óbito infantil no Brasil. 


\section{Resumo}

Os óbitos de menores de um ano foram classificados em causas evitáveis, mal definidas e não evitáveis empregando a Lista Brasileira de Mortes Evitáveis, entre 1997-2006. Foram calculados tendências dos coeficientes de mortalidade infantil por causas de morte e se usou regressão não linear para avaliação de tendência. As causas evitáveis e as causas mal definidas apresentaram significativa redução $(p<0,001)$. As causas reduzíveis de mortalidade apresentaram redução de 37\%. A mortalidade por causas reduzíveis por adequada atenção ao parto declinou em 27,7\%; adequada atenção ao recém-nascido, 42,5\%; e por adequada atenção à gestação cresceu 28,3\%. Concluiu-se que os serviços de saúde contribuíram para a redução da mortalidade infantil. O declínio das causas mal definidas de morte indica ampliação do acesso aos serviços de saúde. O aumento do acesso e atenção ao parto e aos cuidados com recém-nascido contribuíram para a redução de óbitos infantis. O aumento da mortalidade por adequada atenção à gestação revela a necessidade de aprimoramento da atenção pré-natal.

Mortalidade Infantil; Causas de Morte; Avaliação de Serviços de Saúde; Sistema Único de Saúde

\section{Referências}

1. Rutstein DD, Berenberg W, Chalmers TC, Fishman AP, Perrin EB, Zuidema GD. Measuring the quality of medical care: second revision of tables of indexes. N Engl J Med 1980; 302:1146.

2. Suárez-Varela MM, Llopis GA, Tejerizo PML. Variations in avoidable mortality in relation to health care resources and urbanization level. J Environ Pathol Toxicol Oncol 1996; 15:149-54.

3. Charlton JRH, Velez R. Some international comparisons of mortality amenable to medical intervention. BMJ 1986; 292:295-301.

4. Holland WW, Fitzgerald AP, Hildrey SJ, Phillips SJ. Heaven can wait. J Public Health Med 1994; 16 : 321-30.

5. Westerling R. Commentary: evaluating avoidable mortality in developing countries - an important issue for public health. Int J Epidemiol 2001; 30:973-5.

6. Malta DC, Duarte EC. Causas de mortes evitáveis por ações efetivas dos serviços de saúde: uma revisão da literatura. Ciênc Saúde Coletiva 2007; 12:765-76.

\section{Colaboradores}

D. C. Malta contribuiu com a concepção e projeto, análise e interpretação dos dados, redação do artigo e revisão crítica relevante do conteúdo intelectual, aprovação final da versão a ser publicada. E. C. Duarte, J. J. C. Escalante e M. F. Almeida participaram da análise e interpretação dos dados, redação, revisão crítica relevante do conteúdo intelectual, aprovação final da versão a ser publicada. L. M. V. Sardinha participou da concepção e projeto, redação, revisão crítica e da aprovação final da versão a ser publicada. E. M. Macário, R. A. Monteiro e O. L. Morais Neto contribuíram com a concepção do projeto, revisão crítica, aprovação final da versão a ser publicada.
7. Habicht JP, Victora CG, Vaughan JP. Evaluation designs for adequacy, plausibility and probability of public health programme performance and impact. Int J Epidemiol 1999; 28:10-8.

8. Lansky S, França E, Leal MC. Mortalidade perinatal e evitabilidade: revisão da literatura. Rev Saúde Pública 2002; 36:759-72.

9. Abreu DMX, César CC, França EB. Relação entre as causa de morte evitáveis por atenção à saúde e a implementação do Sistema Único de Saúde. Rev Panam Salud Pública 2007; 21:282-91.

10. Malta DC, Duarte EC, Almeida MF, Dias MAS, Morais Neto OL, Moura L, et al. Lista de causas de mortes evitáveis por intervenções do Sistema Único de Saúde. Epidemiol Serv Saúde 2007; 16:233-44.

11. Ortiz LP. Agrupamento das causas evitáveis de morte dos menores de um ano segundo critério de evitabilidade das doenças. São Paulo: Fundação Seade; 2000 
12. Tobias M, Jackson G. Avoidable mortality in New Zealand, 1981-97. Aust N Z J Public Health 2001; 25:12-20.

13. Morgenstern H. Ecologic studies in epidemiology: concepts, principles, and methods. Annu Rev Public Health 1995; 16:61-81.

14. Secretaria de Vigilância à Saúde, Ministério da Saúde. Saúde Brasil 2007: uma análise da situação de saúde. Brasília: Ministério da Saúde; 2007.

15. Macinko J, Guanais FC, Souza MF. Evaluation of the impact of the Family Health Program on infant mortality in Brazil, 1990-2002. J Epidemiol Community Health 2006; 60:13-9.

16. Simões CCS, coordenador. Evolução e perspectivas da mortalidade infantil no Brasil. Rio de Janeiro: Instituto Brasileiro de Geografia e Estatística; 1999.

17. Costa MCN, Mota ELA, Paim JS, Silva LMV, Teixeira MG, Mendes CMC. Mortalidade infantil no Brasil em períodos recentes de crise econômica. Rev Saúde Pública 2005; 39:366-75.

18. Mackenbach JP, Bouvier-Colle MH, Jougla E. Avoidable mortality and health services: a review of aggregate data studies. J Epidemiol Community Health 1989; 44:106-11.

19. Mello-Jorge MHP, Gotlieb SLD, Soboll MLMS, Almeida MF, Latorre MRDO. Avaliação do sistema de informação sobre nascidos vivos e o uso de seus dados em epidemiologia e estatísticas de saúde. Rev Saúde Pública 1993; 27:1-46.

20. Instituto Brasileiro de Geografia e Estatística. Pesquisa Nacional por Amostra de Domicílios: síntese de indicadores - 2003. http://www.ibge.gov.br/ho me/estatistica/populacao/trabalhoerendimento/ pnad2003/sintesepnad2003.pdf (acessado em 13/ Mai/2006).

21. Poikolainen K, Eskola J. Health services resources and their relation to mortality from causes amenable to health care intervention: a cross sectional study. Int J Epidemiol 1988; 17:86-9.

22. Comissão Nacional sobre Determinantes Sociais da Saúde. As causas sociais das iniqüidades em saúde no Brasil. Relatório final. http://www.cndss. fiocruz.br/pdf/home/relatorio.pdf (acessado em Abr/2008).

23. Victora CG. Intervenções para reduzir a mortalidade infantil pré-escolar e materna no Brasil. Rev Bras Epidemiol 2001; 4:3-69.
24. Sansigolo LRF, Pontes K, Miná DL, Barreto ML. Mortalidade infantil e condições sociodemográficas no Ceará, em 1991 e 2000. Rev Saúde Pública 2003; 37:699-706

25. Almeida MF, Alencar GP, Novaes MHD, França Júnior I, Siqueira AA, Schoeps D, et al. Partos domiciliares acidentais na região Sul do Município de São Paulo. Rev Saúde Pública 2005; 39:366-75.

26. Ministério da Saúde. Pesquisa Nacional de Demografia e Saúde da Criança e da Mulher. PNDS 2006. Relatório final. http://bvsms.saude.gov.br/bvs/ pnds/img/relatorio_final_pnds2006.pdf (acessado em 15/Nov/2009).

27. Schoeps D, Almeida MF, Alencar GP, França Jr. I, Novaes HMD, Siqueira AAF, et al. Fatores de risco para mortalidade neonatal precoce. Rev Saúde Pública 2007; 41:1013-22.

28. Barros FC, Victora CG, Barros AJD, Santos IS, Albernaz E, Matijasevich A, et al. The challenge of reducing neonatal mortality in middle-income countries: findings from three Brazilian birth cohorts in 1982, 1993, and 2004. Lancet 2005; 365:847-54.

29. Almeida SDM, Barros MBA. Atenção à saúde e mortalidade neonatal: estudo caso-controle realizado em Campinas, SP. Rev Bras Epidemiol 2004; 7:22-35.

30. Goldani MZ, Barbieri MA, Rona RJ, Silva AAM, Bettiol HJ. Increasing pre-term and low-birthweight rates over time and their impact on infant mortality in south-east Brazil. J Biosoc Sci 2004; 36:177-88.

31. Bettiol H, Rona RJ, Chinn S, Goldani M, Barbieri MA. Factors associated with preterm births in Southeast Brazil: a comparison of two birth cohorts born 15 years apart. Paediatr Perinat Epidemiol 2000; 14:30-8.

32. Bettiol H, Barbieri MA, Gomes UA, Andréa M, Goldani MZ, Ribeiro ERO. Saúde perinatal: metodologia e características da população estudada. Rev Saúde Pública 1998; 32:18-28.

33. Silveira MF, Santos IS, Matijasevich A, Malta DC, Duarte EC. Nascimentos pré-termo no Brasil entre 1994 e 2005 conforme o Sistema de Informações sobre Nascidos Vivos (SINASC). Cad Saúde Pública 2009; 25:1267-75.

34. Nolte E, McKee M. Does health care save lives? Avoidable mortality revisited. London: Nuffield Trust; 2004 .

Recebido em 24/Ago/2009

Versão final reapresentada em 10/Dez/2009

Aprovado em 18/Jan/2010 